\title{
Initiating the 2002 Mars Science Laboratory (MSL) Focused Technology Program ${ }^{12}$
}

\author{
Robert T. Caffrey \\ NASA / Goddard Space Flight Center \\ Greenbelt, MD 20771 \\ robert.t.caffrey@nasa.gov \\ Gabriel Udomkesmalee \\ Jet Propulsion Laboratory \\ California Institute of Technology \\ Pasadena, CA 91109 \\ suraphol.udomkesmalee@jpl.nasa.gov \\ Samad A. Hayati \\ Jet Propulsion Laboratory \\ California Institute of Technology \\ Pasadena, CA 91109 \\ samad.hayati@jpl.nasa.gov \\ TABLE OF CONTENTS
1. INTRODUCTION
2. MSL TECHNOLOGY OVERVIEW
3. TECHNOLOGY PROGRAM IMPLEMENTATION
4. TECHNOLOGY MANAGEMENT TOOLS
5. CONCLUSION
6. REFERENCES
7. ACKNOWLEDGMENTS \\ 8. BIOGRAPHIES
}

\begin{abstract}
The Mars Science Laboratory (MSL) Project is an aggressive mission launching in 2009 to deliver a new generation of rover safely to the surface of Mars and conduct comprehensive in situ investigations using a new generation of instruments. This system will be designed to land with precision and be capable of operating over a large percentage on the surface of Mars. It will have capabilities that will support NASA's scientific goals into the next decade of exploration. The MSL Tcchnology Program is developing a wide-range of technologies needed for this Mission and potentially other space missions. The MSL Technology Program reports to both the MSL Project and the Mars Technology Program (MTP). The dual reporting process creates a challenging management situation, but ensures the new technology meets both the specific MSL requirements and the broader Mars Program requirements.
\end{abstract}

MTP is a NASA-wide technology development program managed by the Jet Propulsion Laboratory (JPL) and is divided into a Focused Program and a Base Program. The Focused Technology Program addresses technologies that are specific and critical to near-term missions, while the Base Technology Program addresses those technologies that are applicable to multiple missions and which can be characterized as longer term, higher risk, and high payoff technologies. The MSL Technology Program is under the Focused Program and is tightly coupled to MSL's mission milestones and deliverables. The technology budget is separate from the flight Project budget, but the technology's requirements and the development process are tightly coordinated with the Project.

The Technology Program combines proven management techniques of flight projects with commercial and academic technology management strategies, to create a technology management program that meets the near-term requirements of MSL and the long-term requirements of MTP. This paper examines the initiation of 2002 MSL Technology program. Some of the areas discussed in this paper include technology definition, task selection, technology management, and technology assessment.

\footnotetext{
${ }^{1}$ U.S. Government work not protected by U.S. copyright

2 IEEEAC paper \# 1473, Updated Jan 19, 2004
}

\section{INTRODUCTION}

The Mars Science Laboratory (MSL) Project is an aggressive mission launching in 2009 to deliver a new generation of rover safely to the surface of Mars and conduct comprehensive in situ investigations using a new generation of instruments. This system will be designed to land with precision and be capable of operating over a large percentage on the surface of Mars. It will have capabilities that will support NASA's scientific goals into the next decade of exploration.

This paper examines our plans at the Jet Propulsion Laboratory (JPL) to define and initiate MSL's Technology Program. When we initiated this Technology Program in 2002 , we were unable to locate guidelines or examples of how other missions in pre-formulation developed their technologies. To address this issue and provide other projects and programs with an example of a technology implementation plan, this paper reviews the detailed processes MSL used to initiate its program. In fact, early versions of this paper were provided to other projects at JPL and the Goddard Space Flight Center (GSFC) to help them develop their technology plans. These projects eventually implemented elements of MSL's Technology Plan.

The Mars Technology Program (MTP) is comprised of a Focused Technology Program and a Base Technology Program [1]. The Focused Technology Program addresses 
technologies that are specific and critical to near-term missions, while the Base Technology Program addresses those technologies that are applicable to multiple missions and which can be characterized as longer term, higher risk, and high payoff technologies. MSL's Technology Program is under the MTP Focused Technology Program and is roughly an $\$ 80$ million effort to develop the technology needed to meet the unique requirements of the MSL Mission.

The MSL mission introduces a number of significant technological innovations to enable the following capabilities: 1) dramatically increased landing precision and accessibility to the Martian surface by landing within a 10kilometer major axis ellipse between $60^{\circ} \mathrm{N}$ and $60^{\circ} \mathrm{S}$ latitudes, and up to +2.5 kilometer altitude, 2) extended mobility range adequate to ensure representative measurement of diverse sites, at scales of several kilometers; with a goal to demonstrate a total traverse distance of greater than $10 \mathrm{~km}, 3$ ) long-lived mobility asset, ensuring operability for a minimum of one Mars year (2 Earth years), and 4) operation of an analytical science laboratory capable of definitive analysis of the mineralogy, chemistry, and isotopic composition of surface and nearsurface materials; including the assessment of biological potential of the landing site. These technology innovations carry forward into future missions in the second decade of this century.

\section{MSL Technology Overview}

MSL is equipped with an onboard science laboratory that performs in-situ analytical experiments. In addition to being a full science mission, MSL will provide feed-forward technologies that are required by future missions to land large payloads and operate for a long time on the surface of Mars. An example of such a future mission is the Mars Sample Return mission [1].

There are two main technology areas that are required for the MSL mission: Entry, Descent, and Landing (EDL) capability and Surface System technologies. EDL technology includes guidance, deceleration, and landing technologies and surface system technology includes rover, long-life, and sample preparation and distribution technologies. The following paragraphs describe these technologies.

Authors note: The information in this paper is based on conceptual designs (at this writing, approximately two years prior to the project preliminary design review (PDR)). Readers should appreciate that actual capabilities of the landed mission may ultimately be less than or greater than described here.

\subsection{Entry, Descent, and Landing (EDL)}

One of the main goals of the MSL technology program is to develop the capability to land safely near any chosen site (including higher elevations) in order to provide a near global access to Mars. To achieve this ability to land safely at any locality on Mars, the entire process of entry, descent and landing was reviewed and technology innovations initiated to provide the needed capabilities. Figure 2-1 shows the EDL technology goals.

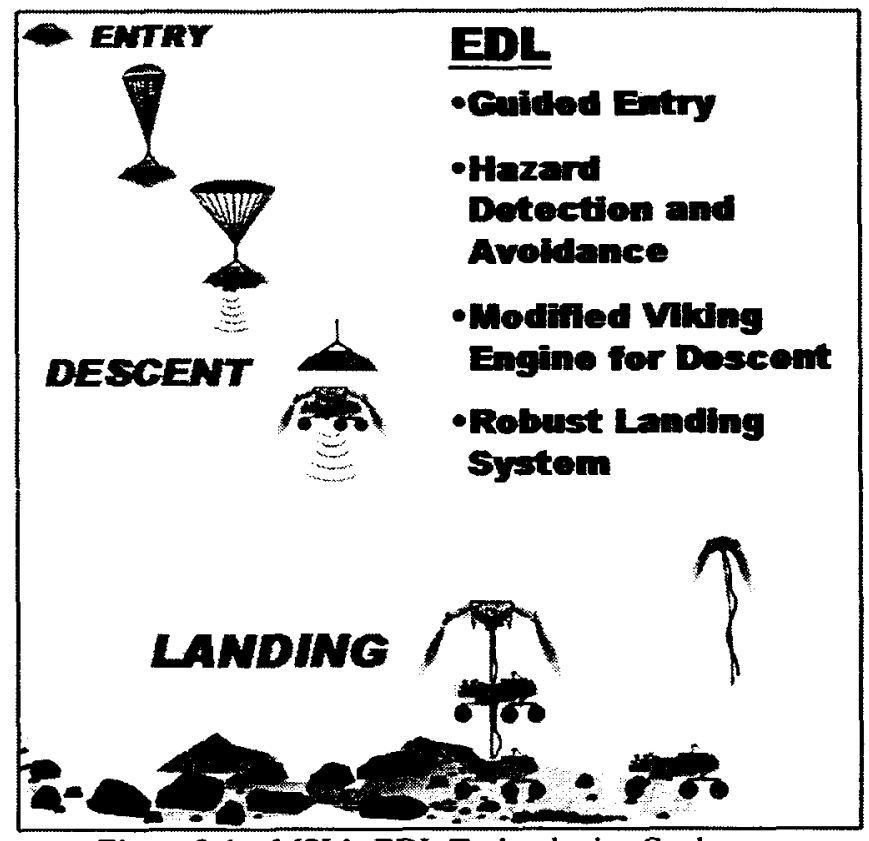

Figure 2-1 - MSL's EDL Technologies Goals.

Past Mars Mission utilized radio-based approach navigation, no control of the lift vector during entry, a single stage parachute, and only limited hazard tolerance (airbags or landing ruts). There was no hazard detection/avoidance capability. Variants of this approach were used on Viking and Pathfinder and are currently in use on the 2003 Mars Exploration Rover (MER) mission. The errors in approach navigation propagate through the trajectory, resulting in a landing error ellipse ranging from $100 \mathrm{~km}$ cross range to $300 \mathrm{~km}$ along the downrange vector. The payload mass that can be delivered using existing or anticipated launch vehicles with this system is limited to $\sim 200 \mathrm{~kg}$.

Precision landing reduces the risk of encountering hazardous areas such as large craters and steep canyons at any given region of Mars where it is desired to land. The goal is to develop new technology to reduce the landing error ellipse to smaller than $5 \times 10 \mathrm{~km}$. In addition the payload mass will be raised to $\sim 900 \mathrm{~kg}$. To achieve this goal, each step of the EDL process is upgraded with new technology. Each step is developed with advanced technology, with systems engineering used to assure that all the pieces fit together. To achieve this goal, three complementary technologies are in development: Precision Landing; Hazard Avoidance; and Robust Landing. 
Precision Landing - A lifting entry vehicle is in development with $\mathrm{L} / \mathrm{D} \sim 0.25$ (lift/drag) that is capable of actively guiding itself from entry to parachute deployment despite uncertainties in the entry conditions, atmospheric density profile, winds and aerodynamic performance. The two-stage parachute system includes a supersonic chute and a subsonic chute. Improved descent propulsion is achieved by recapturing Viking technology with new improved components.

Hazard Detection and Avoidance - Hazard avoidance provides an "eyes wide open" capability at landing. The overall process is illustrated in Figure 2-1. This makes it possible to make adjustments to the landing location to avoid large craters, large rocks and steep local slopes. MSL is developing an integrated terminal guidance, navigation, and control system that predicts the descent flight path after the subsonic parachute is deployed, detects hazards such as rocks and slopes using active sensors, and after jettisoning the parachute, steers the lander to a safe landing area. A RADAR sensing system for 0-7 km altitudes is in development to generate initial terrain maps at $500 \mathrm{~m}$ altitude and create initial position estimates of the landing area at $\sim 7 \mathrm{~km}$ altitude. This system provides more time for the lander to be laterally maneuvered to avoid undesirable landing sites.

Robust Landing - Once landing is committed to, this technology makes it possible to tolerate the unexpected and provide resilience to failures in either precision landing or hazard avoidance systems. The baseline plan is to get away from the landing pallet system and all its mass, egress, and design problems and develop a sky-crane system that is less complex and provides better technology feed forward capability (for Mars Sample Retum).

\subsection{Surface System Technologies}

These technologies include all the technologies that enable a rover to travel long distances, place instruments autonomously, and analyze samples in its onboard laboratory. The rover and its instrumentation must last at least one Martian year.

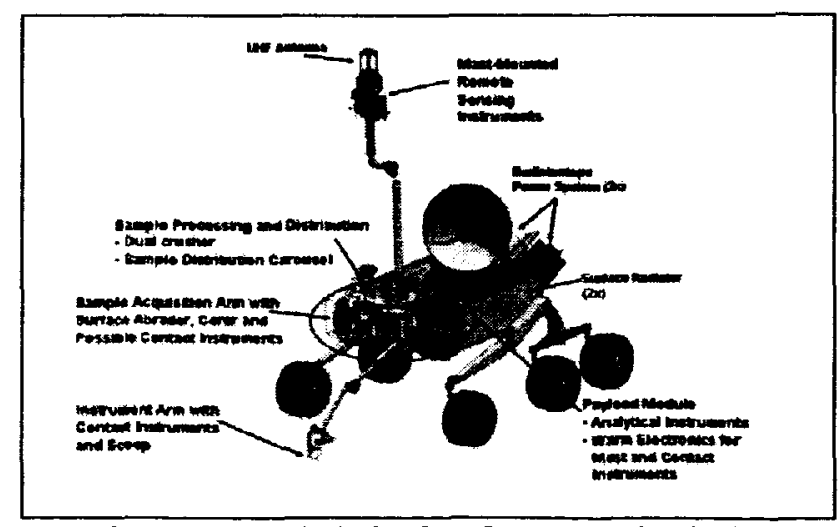

Figure 2-2 - MSL's Surface System Technologies
Improved rover autonomy is developed to enable safe navigation as well as autonomous science operations for Mars surface missions. The strategy is to integrate technologies from MTP's Base Program that are competitively selected and integrate them to demonstrate the capabilities that are required for MSL.

Long Life - A study was conducted to determine which of the rover components are likely to fail during the one Martian year mission and it revealed that there are major weaknesses in the area of mechanical systems, particularly motors and bearings. Another major area was the electronics which would be exposed to the extreme Martian temperature fluctuation. MSL has a Thermal Cycle Resistant Electronics (TCRE) task to improve and test potential hardware under realistic conditions.

Sample Processing and Distribution Experiment (SPADE) One of the main differences between MSL and past Mars missions is MSL's capability to analyze Martian samples onboard its analytical laboratory. The laboratory consists of science instruments, sample acquisition, processing, and distribution system.

The objective of the SPADE system is to crush rocks (0.1-1 $\mathrm{mm}$ ) and distribute them to various onboard science instruments. Cross contamination for this type of a system is a major concern. A technology requirement has been established to insure that no more than $5 \%$ contamination results from one sample to the next. This number can be decreased by processing the second sample more than once, thus reducing the contamination to levels below $5 \%$.

The previous paragraphs provided an overview of the technologies needed for the MSL Mission. The following sections describe how MSL and MTP defined, selected, and managed the specific technologies need by MSL.

\section{TECHNOLOGY PROGRAM IMPLEMENTATION}

The purpose of the MSL Technology Program is to retire technical risks while the project is in formulation and before it enters implementation. If a technology risk cannot be retired before implementation, the project still has time to select another technology and modify their mission concept. The technology program matures the most effective technologies while balancing each technology's performance, cost, risk, and schedule. During the technology program, MSL matures the required technologies from their present Technology Readiness Level (TRL) that is typically between TRL 2 and 3 to a TRL of 6 .

TRLs are a systematic metric/measurement system that supports assessments of the maturity of a space technology and provides a method of comparing maturity between different types of technology. The TRL scale is from 1 to 9 , 
where 1 represents a technology principle and 9 represents a technology flown successfully in space. Projects that are not technology demonstration missions typically don't accept the risk of including a technology with a TRL of less than 6 in their design concept. TRL 6 corresponds to a technology functioning in a subsystem (model or prototype) in a relevant environment (ground or space).

NASA's guidelines for Project Management, NPG 7120.5B, states:

The purpose of the formulation subprocess is to define a project concept and plan for implementation to meet mission objectives... the formulation subprocess ... assesses the technology requirements and develops the plans for achieving the technology options ... [2]
The following sections describe how MSL defined its project concept, assessed its technology requirements, and developed its technology implementation plan.

MSL's technology implementation process, show in Figure 3-1, include three major stages: technology definition, technology task selection, and technology management. The following sections describe each of these efforts and relate them back to Figure 3-1. Figure 3-1, MSL Technology Implementation Plan, also lists the paragraph numbers of the following sections to show how the flow of the paper corresponds to the flow of the technology implementation process.

The MSL Technology Program is complete when it technology matures to TRL 6. The goal of the MSL Project is for all its technology to reach TRL 6 by the Mission Preliminary Design Review (PDR) in 2005.

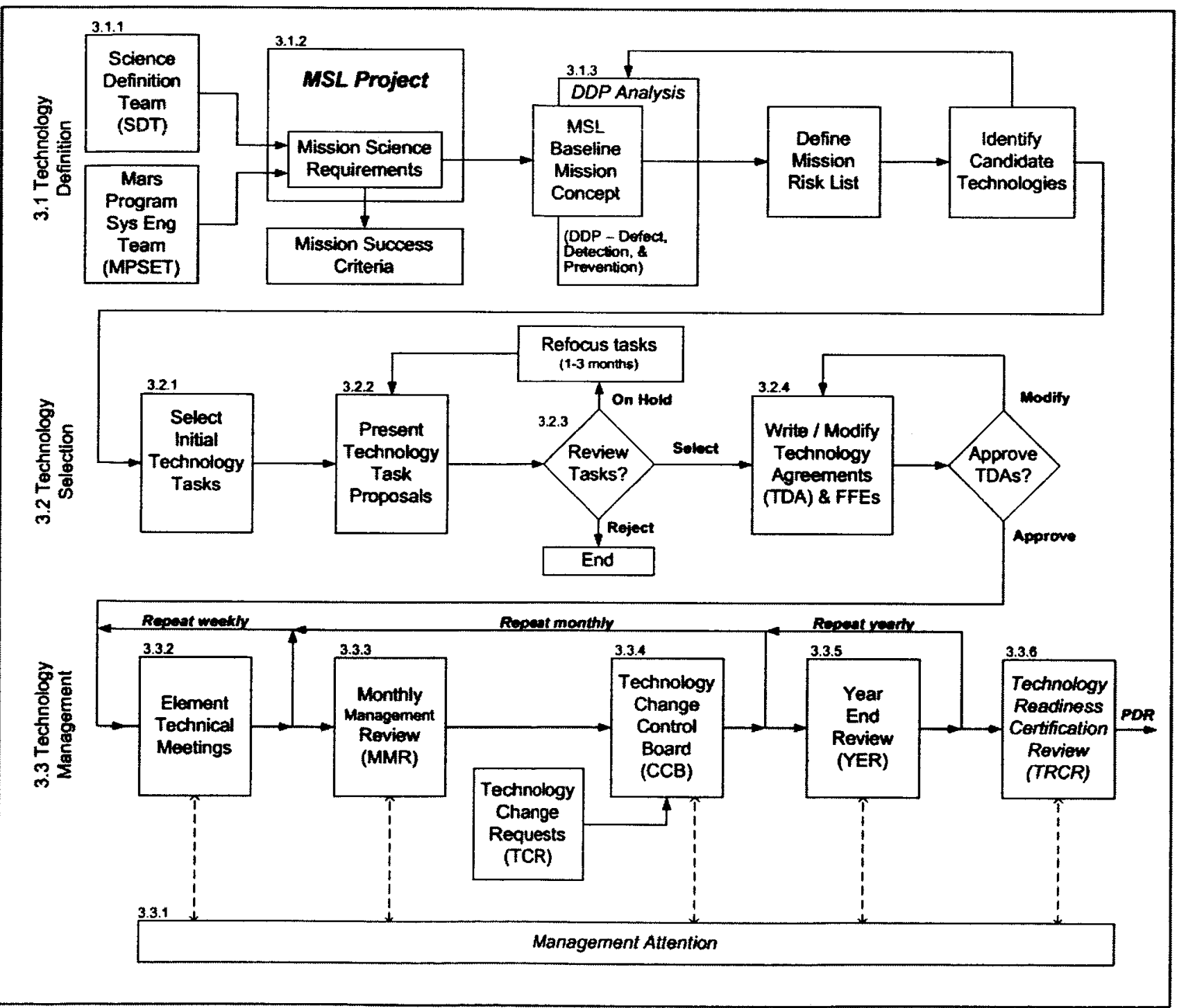

Figure 3-1 - MSL Technology Implementation Process 


\subsection{Technology Definition}

The first stage of MSL's technology implementation process is Technology Definition. In this stage, as described below, the different organizations work together and produce science requirements, a mission concept, a mission risk list, and a list of candidate technologies that reduce the mission risks.

\subsubsection{Development Organization - Central to} implementing a successful technology program is an effective organization structure. The following section describes the 2002 MSL organization (shown in Figure 3-2) that defined and initiated the MSL technology program. The positions in this organization chart will be referred to as the implementation process is described. The roles and responsibilities of these positions are described below.

At the top and center of this chart are the Science Definition Team (SDT) and the Mars Program System Engineering Team (MPSET). The SDT defines and prioritizes the science goals, objectives, investigations, and measurements of MSL. The SDT ensures that the MSL science requirements and mission concept are consistent with Mars Program science themes. The MPSET ensures that the Mars Program is optimized as a whole and not on a basis of project by project. MPSET also works with the Mars
Technology Program (MTP) and constituent projects to maximize the program's technology readiness. If a technology is needed for multiple missions, MPSET ensures that the requirements of both missions are included in the initial development effort. For example, an earlier Mars mission (MRO) developed an optical navigation (OpNav) camera and included the requirements of both the MRO and MSL missions in the development process. The minimal cost impact to MRO is justified by the significant benefit to MSL and the Mars Program.

An interesting feature of the MSL Technology Program is the dual reporting structure. The MSL Focused Technology Manager reports to both the MSL Project Manager and the MTP Program Manager. The MSL Project Manager ensures technology meets the MSL focused requirements and the MTP Program Manager ensures technology meets the longrange requirements of the Mars Program. This inherent conflict actually benefits the project and the program by providing a checks-and-balance to many budget and technical decisions.

Five Element Managers report to the MSL Technology Manager. The Element Managers are responsible for major systems in the project and ensure that the technologies underdeveloped are compatible with the latest mission concept. They also ensure the mission concept is consistent with the progress of the different technology tasks.

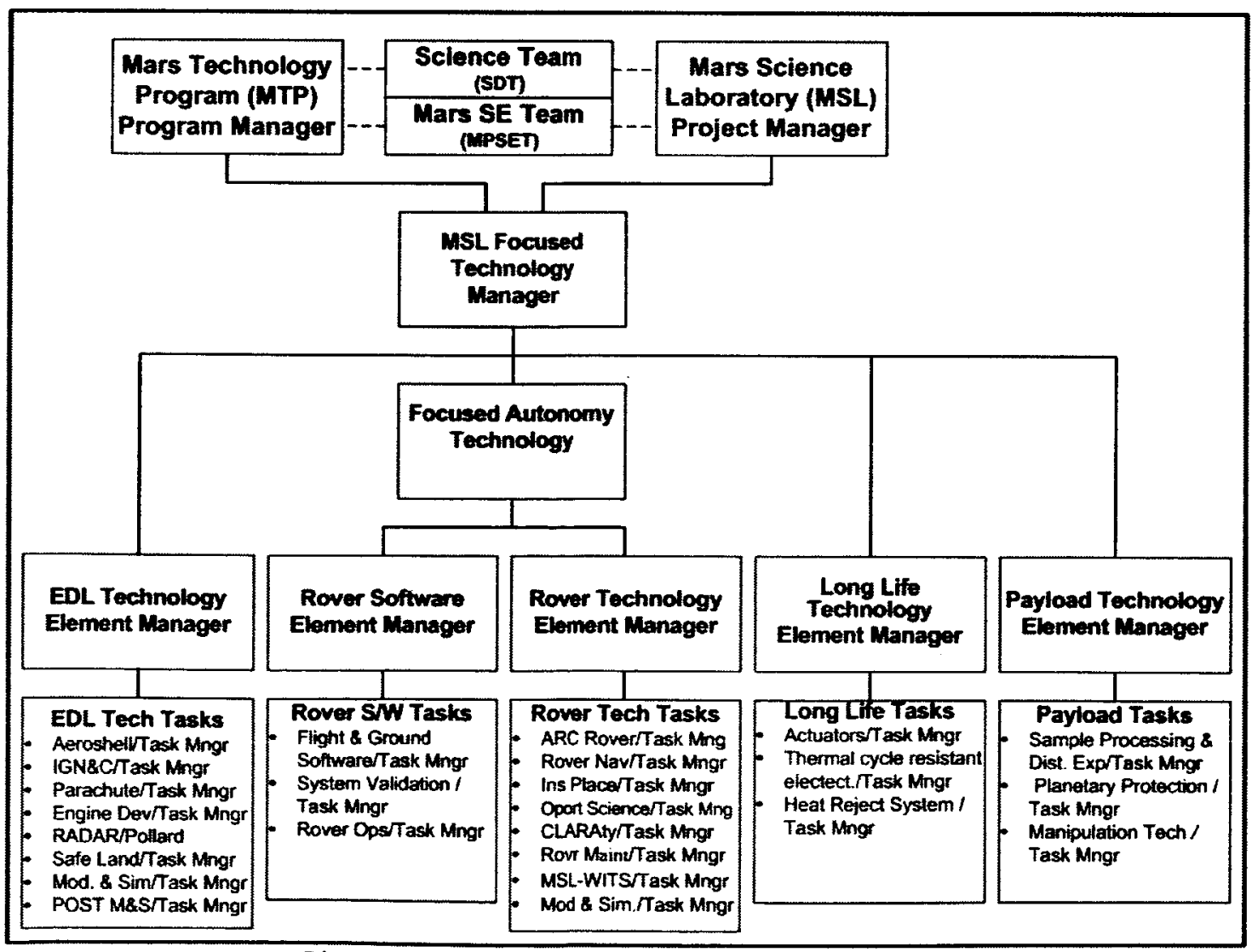

Figure 3-2 - 2002 MSL Technology Organization Chart 
3.1.2 Science Requirements and Mission Concept - The first step in technology implementation (Figure 3-1) is to define the technology to be developed. This may sound obvious and simple, but in a large organization with competing views, issues, and priorities, agreeing on technology requirements is a challenging task.

On MSL the process began with the project working with both the MPSET and the SDT to develop the mission science requirements (Level 1). Next, the project manager, element managers, and different working groups transformed these requirements into a baseline mission concept (where 'baseline' implies the concept will change as the both the technology and the project definition matures). The project uses the baseline mission concept to generate the system-level performance requirements. The following sections concentrate on the process of converting the mission concept into an effective technology implementation plan.

3.1.3 Mapping Requirements to Technology - The technology definition process continues by reviewing the mission concept and the system-level performance requirements in informal (peer) reviews, formal reviews, and working group meetings. These meetings defined the major risks and identify candidate technologies to reduce these risks. These risks include performance risks, costs risks, and schedule risks. Through MSL's technology definition and selection process, the project initiated technology tasks that reduced risk in all three areas.

MSL used a process for managing these risks called Defect Detection and Prevention (DDP) [3]. The DDP process has three steps: 1) determine where we want to be, 2) identify what could get in the way, and 3) decide how we will get there. MSL implemented the DDP steps and put their system-level performance requirements in a tree structure to 'determine where we want to be' and captured as trees of potential failure modes 'what could get in the way'. MSL then scored the impacts of these failure modes on the science requirements to prioritize the failure modes. From the list of failure modes, MSL created their initial Mission Risk List.

The third step, 'decide how we will get there', is probably the most challenging and requires the most iterations with the project, program, and cost modeling organizations. Technology investments were assigned to each risk area and the DDP tool captured the estimate of how each risk area was reduced. A 'top down' approach, based on cost models and project experience, was used to estimate the cost of the different technology investments. A 'bottom up' cost estimate is done after a task manager is assigned and this process is examined in a following section.

Each technology investment, constrained by the technology budget, addressed an item on the prioritized risk list. The risks, and their corresponding technology, include performance risk, schedule risk, or cost risk. Examples of the three types of risk and their corresponding technology are as follows: 1) existing engines do not meet MSL performance requirements so we invested in new engines, 2) current mission operations do not meet MSL's extended life requirements so we invested in improved operations and science collaboration; and 3) the cost (in dollars and mass) to heat external electronics is high so we invested in temperature extreme electronics.

The process to determine which combination of technologies minimize mission risk and should be funded is described in the following sections.

\subsection{Technology Selection}

The second stage of MSL's technology implementation process is Technology Selection. This stage started with a list of candidate technologies and produced detailed implementation plans for each technology task. The MSL technology selection process took five months for most of its tasks and an additional four months to refocus and select the remaining tasks. The Technology Selection process is examined in the following paragraphs.

3.2.1 Assign Task Managers - After MSL agreed to a candidate list of technologies, the Mars Program, the MSL Project, Element Managers, and functional managers together selected and approved Task Managers to lead each development effort. Task Managers are typically technologists with mixed levels of flight project experience. Since MSL is a focused technology effort, these tasks managers became integral members of the project team. Experienced Element Managers work with each Task Managers to ensure projects requirements are meet. The tightly coupled technology/project environment required the technologist to be less autonomous and more driven by project cost, schedule, and performance constrains. This initially was challenging for technologists accustomed to working independently, but the common goal of a successful planetary mission helped unify the diverse team members.

\subsubsection{Prepare Task Proposals - Using the baseline mission concept and the system-level performance requirements, each task manager worked with their element manager to develop a presentation that proposed how their task would meet the mission requirements. These proposals were presented to the MTP Program Manager and the MSL Project Team. The presentations described how the task would improve the current state-of-the-art, for what cost, and in what schedule. The following is a detailed list of what each presentation included and why it was requested. As stated earlier, this detail is not needed to understand the MSL Technology Program, but it may be useful to another project initiating their technology program.}


Overview (Quad Chart) - A one slide summary of the proposed task including a representative picture, objectives summary, milestones list, organizations involved, collaborators, outside companies, and a subtask multi-year schedule including funding levels. This chart is not frequently updated, but it is useful for someone unfamiliar with the task to get a summary of the technology.

Objectives and approach - These slides described the overall task objectives, quantify the current state of the art, and quantify the new performance goals. They also explained the implementation approach and provide a cost summary. In addition, the task's milestones (or accomplishments) are listed. These charts provide insight to the reviewers on the task manager's grasp of the problem, plan to solve the problem, and the metrics to show progress.

Backup data - Included a list of questions to help the reviews assess the impact, risks, and procurements associated the task. Question 1: What is the functional relationship between the technology task and the mission goals? This question ensures that the task address a critical need of the project and is not just an interesting technology effort. Question 2: What are the assumptions, dependencies, and risks associated with the task? This question identifies any issues with the task. Question 3: Explain any outside procurements? This question identifies if the task needs a large procurement. Procurements are complex, so identifying them early can avoid future problems.

Receivable/Deliverable Lists - List all the items being received by the task and delivered from the task to a project element. A significant difference between a focused technology task and a base technology task is the tight coupling of the technology task to the flight project. A flight project is driven by its launch date and other key milestones. A technology task must synchronized its deliverables and schedule to the flight schedule. Each technology task must closely track what items it expects from a flight system or another technology task. A problem in one task could ripple through multiple tasks and flight systems, so the project must track the dependencies.

3.2.3 Technology Tasks Review - Approximately thirty technology tasks were presented to the MSL Project and the MTP Program in a three step process: project reviews, a functional organization review, and a program review. The project reviews included three different reviews over a period of two weeks. Each review refined the technology implementation concept and helped communicate the project's requirements and mission concept.

The second step in the task review process was the JPL functional organization review. This meeting gave the functional managers a chance to review what commitments the task managers were making of their organizations. It also provided a forum for the project and the functional organizations to discuss the latest technology requirements and mission concept.

The third step in the task review process was the four day MTP Year End Review (YER). The Mars Technology Program (MTP) conducted this meeting and included reviewers internal and external to JPL and the Mars Program. They provided an independent assessment of the MSL technologies with an eye on the big picture. The independent reviewers are aware of technology development efforts at other government, university, and industry organizations and frequently recommended MSL technologists talk with other organizations. This helped optimize the Focused and Based Technology Programs.

The task review process resulted in three categories of tasks: selected, rejected, and tasks on hold. Most tasks were selected, but the review process modified most implementation plans by changing their budget, development schedules, and development process. Some tasks were rejected because their modest improvements in the state-of-the-art did not justify their budget. For example, one task proposed reducing the size and mass of a flight system. However, the small improvement in size and mass did not warrant the cost and risk of the task.

Several task plans lacked the necessary details and these tasks were put on hold until they refocused their plans. Most of these tasks received a low level of funding while they refined their concept and created new implementation plans. These tasks were eventually funded, but some with a dramatically different scope and implementation plan.

\subsubsection{Technology Development Agreements (TDA) -} Once a technology task was selected, task managers and their element managers developed Technology Development Agreements (TDA). A TDA is a roadmap of the process to mature a technology from its current TRL to TRL 6. They also contain specific test protocols for each TRL transition as well as the associated costs, schedules, and facilities to achieve each TRL transition. These Technology Development Agreements (TDA) and their related test protocols represent a process that is performance-oriented and paced by the TRL transitions. To maximize the likelihood of meeting the overall schedule and budget, each technology task was assigned reserve funds to cover unforeseen problems. Reserves were assigned by the MTP Program Manager and the MSL Element Managers based on the risks of each tasks. The reserves are reviewed at the monthly management reviews (MMR), which are described later in this paper.

A TDA is a web-based technology management tool that captures the technical and programmatic information related to a technology task. NASA Headquarters requires JPL and other NASA Centers to complete an annual technology inventory. TDAs capture the information required by this technology inventory. 
The following is a detailed list of what is in a TDA and why it was requested. As stated earlier, this detail is not needed to understand the MSL Technology Program, but it may be useful to another project initiating their technology program.

Introduction - Describes the technology, assesses the stateof-the-art, and defines the current TRL.

Objectives - Describes the task's technical objectives and goals. In addition, this field defines the specific mission requirements this task will enable. This section documents how a task meets mission requirements.

Technical Approach - Describes the methodology and approaches to conduct the proposed development. Define the products and/or expected results. Provides information on technology development approaches such as analysis, experiment, field testing, and, if applicable, flight validation. This field provides reviewers with an understanding of how a task will be implemented.

Significance - Explains how the task will contribute to a NASA Mission. Examples are mission enabling, mission enhancing, increasing safety margin, reducing mass, lowering mission cost, etc. Mention possible applications to other missions. Describes the technology's current performance level and what level of performance the new technology will achieve. This field ensures the task is tied to a mission requirement and not simply an interesting technology development effort.

Milestones and Deliverables - Identifies the dates when major milestones will be achieved. At least three milestones are required in each fiscal year of the task. The list includes events, dates, and descriptions. This field provides the metrics that will measure the progress of the task.

Funding Distribution - Lists the type of budget elements (people, parts, etc), who is being funded, which fiscal year, the yearly totals, and the total to complete. This field is completed after the task manager completes the budget estimate tool (Friendly Front End, FFE). The FFE tool is described later in this section.

Documented Partnerships/Cooperative Agreements Describes any formal partnerships, cooperative agreements, or other agreements that involve this task. However, proprietary or partner sensitive information are not to be included in this field.

Comments - The following questions determine the tasks funding sensitivity, work breakdown, task dependencies, and other issues associated with the task: 1) What is the impact if funding is increased by $20 \%$ and decreased by $20 \%$ ? 2) What are the priorities of the different sub-tasks? 3) What is the task's probably of success and the backup plan if not successful? 4) How is this technology dependent on other development efforts? 5) Can the technology scale for different configurations and what is the impact on mass and power? 6) What are the technology's interfaces and are they standard? 7) Describe any out-of-house efforts related to this task and at what level of funding. 8) What procurements are planned and what is the acquisition plan?

Infusion Plan - Defines the plan for applying the technology developed in this task to a practical implementation. Final implementation includes either a flight project or a ground application. Unlike base technologies, the infusion plans for MSL's focus technologies, are driven by the element managers, but this field captures the initial plan.

Reporting Plan - Defines the plan for reporting status/progress on this task to management. This should include task reviews, non-advocate reviews, project review, written reports and publications. Again, MSL drives the reporting plan for its focused technologies, but this field lets task managers know what to expect in the future.

Commercialization Plan - Defines the plan for transferring the technology developed in this task to commercial use. If appropriate, this field identifies the industry partner teaming on the commercialization effort.

Approval - The TDA sequence of approval is: Task Manager, Element Manager (or Level-1 Manager), Section Manager, and Project Office. Other members of the project team, including members of the science team, review the technologies at the initial proposal review and the periodic status reviews.

After the TDA is approved, the task implements their technology development plan. The TDA is moved under Configuration Management (CM) control and any future changes require approval from MSL's Change Control Board (CCB). The MSL CCB is discussed later in this section. TDAs are reviewed at the end of every fiscal year and changes are made to reflect the technology development updates for the next fiscal year.

3.2.5 Technology Budget Worksheet (FFE) - Each task is given a fixed amount of money to develop its technology. The TDA captures the cost estimate to complete the task including purchases, travel, subcontracts, and manpower. The data for these fields are generated by the Friendly Front End (FFE), a financial spreadsheet tool that collects all the budget information for the technology development task. The FFE permits detailed cost estimates with the rates and factors are incorporated into the software. The FFE estimates: workforce, procurements / subcontracts, and travel / services.

Every month, MSL's Project Resource Analysts (PRA) generates financial reports for each technology development task and each Project Element. These reports list the actual spending, compare the actual costs to the planned budget, and flag any variances. If a technology development task is 
under-running or overrunning, the task manager must explain the discrepancy at the next monthly management review (MMR) and assess the impact to both the technology task and the MSL Project.

\subsection{Technology Management}

The third stage of MSL's technology implementation process is Technology Management. This stage begins when the technology tasks are approved and continues until the tasks are completed. A task is completed when it reaches a Technology Readiness Level (TRL) of 6, which typically requires three years. Each task manager defines how their technology will reach TRL 6 (technology demonstrated in a relevant ground or space environment).

3.3.1 Management Attention - A number of research studies show increased management attention improves the performance of an effort and increases the likelihood of success. The following paragraphs examine two such studies and describe how we applied these studies to our technology program.

Timing and Impact of Management Attention - Hayes, Wheelwright, and Clark [4,5] examine the relationship between the timing of management attention and the ability to influence a project's outcome. The results of this research, shown in Figure 3-3, highlight that organizations typically devote management attention to a product late in its development cycle when it is difficult to influence the outcome. Management traditionally concentrates on projects that are closest to their launch date, whether it is a product launch or a satellite launch. However, the closer to a launch date the more difficult and expensive it is to make changes.

On MSL, we devoted considerable management attention to the technology program earlier in its development cycle to more effectively influence its outcome. We conducted initial project, program, functional, and independent reviews. We also held regular element, project, and program reviews. In addition, we conducted frequent oneon-one technology manger and task manager discussions to better understand technology issues, constraints, and dependencies. The periodic reviews are described later in this section.

Frequently technologists are left alone in a lab to develop their technology and are not engaged until their deliver date approaches. Some technologists like this independence, but on MSL many of the technologists appreciated being more involved in the day-to-day activity of a planetary mission. MSL, with its tight budget and schedule, could not wait for the delivery of a technology and hope it fit into their system design, so we were significantly involved in the early phases of technology development.

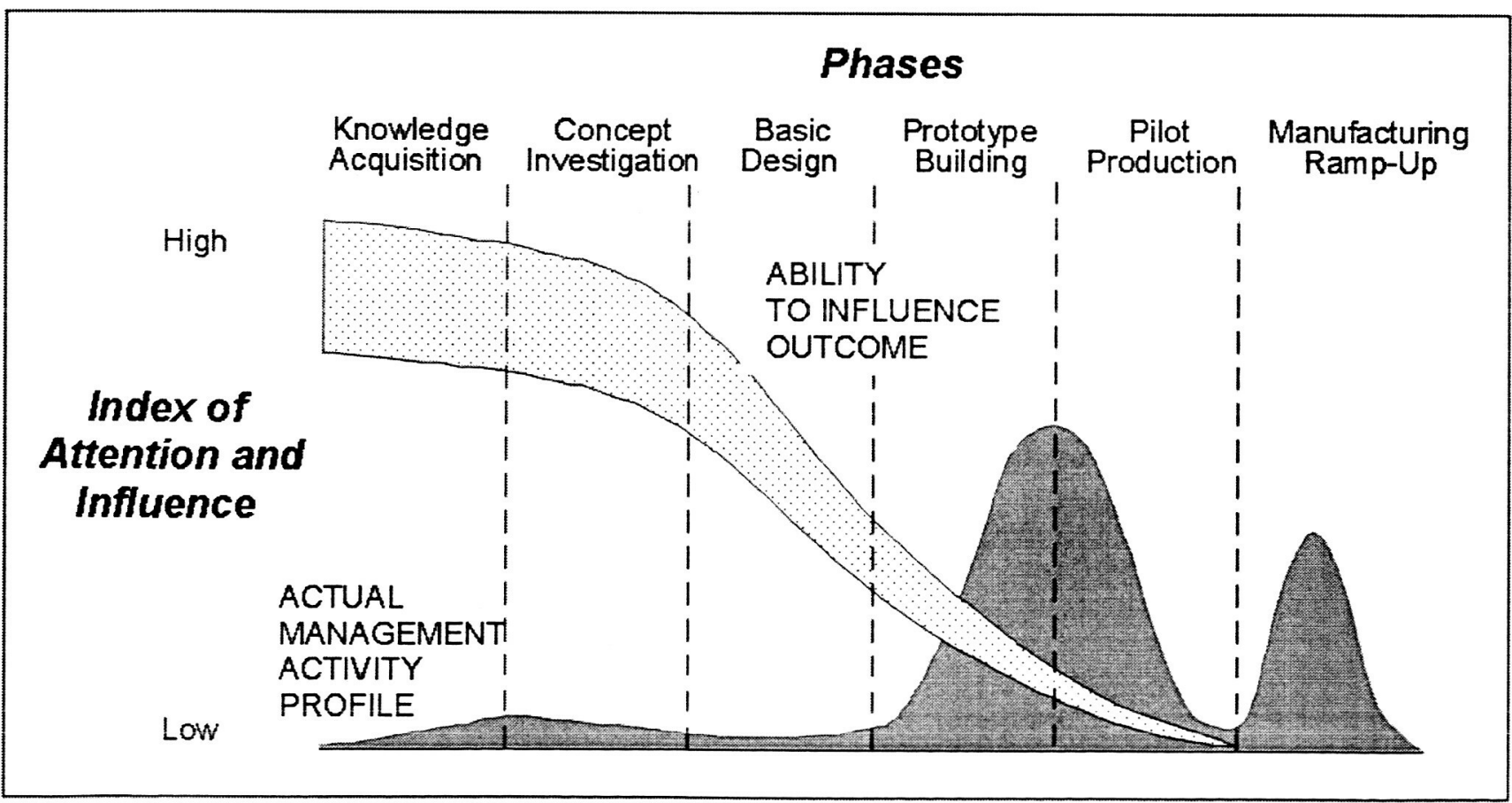

Figure 3-3 - The Timing and Impact of Management Attention and Influence [4] 
Hawthorn Effect - In a research project (1927 - 1932) at the Hawthorne Plant of the Western Electric Company in Cicero, Illinois, Harvard researchers (Mayo, Roethlisberger, and Dickson) discoved that process operators, who knew their process performance was being measured, actually performed better [6]. One conclusion from the study was that workers were pleased to receive attention from the researchers who expressed an interest in them. On MSL, technologists frequently expressed gratitude with our interest in their technology development. MSL is one of the flagship missions at JPL and technologists appreciated being more involved in this exciting mission to Mars.

On MSL, we measured the performance of technologists through weekly and monthly reviews (discussed in the following sections). We paid close attention to accomplishments, budgets, and deliverables. Asking technologists to report progress periodically encouraged them to have something good to report and helped drive productivity.

\subsubsection{Weekly Element Meetings - The Element Managers} are responsible for organizing and conducting weekly technical meetings of the technologies in their element. The appropriate task managers and system engineers support these weekly technical meetings. These meetings discuss the technical progress of the technology tasks, examine the dependencies of the different tasks, and review both the impact of the technology on the project's concept and the impact of the maturing project concept on the technology.

During the program, the Element Managers track the progress of both the mission concept and the technology development efforts. As the mission concept changes, the technology requirements are updated, and as the technology effort makes progress, the mission concept is updated.

3.3.3 Monthly Management Reviews (MMR) - The MSL Technology MMR is organized and conducted by the MSL Project Manager and usually proceeds the MSL Project MMR. The MMRs are supported by the line organizations, the Program Office, and collaborating NASA centers. The MMR reviews technical progress (planned v. actual), action items, issues, budgets, schedules, and deliverables. Reserves, Risk Lists, Lien Lists, and other status charts are also reviewed at the MMRs.

The MMR is supported by the project and the functional organizations, but every quarter the MMR is opened to a broader audience. These Quarterly Reviews replace that month's MMR. The Element Managers prepare and present at the Quarterly Reviews for their respective technical areas. The participants include MSL Project staff, NASA Program Executives, representatives from collaborating NASA Centers, line management, and technology customers (project and pre-project managers).
3.3.4 Technology Change Process - As the MSL technology tasks proceed and as the MSL Project concept matures, changes to the technology tasks are inevitable. In addition, the MSL technology program is managed by both the MSL Project, and the MTP Program, each with a slightly different focus. MSL desires to optimize the project and MTP desires to optimize the program. The technology change control process (shown in Figure 3-4) involves all impacted parties in the change process and balances the healthy tension between the MSL Project and the MTP Program.

The CCB meetings are convened on an as-needed basis by the Technology Manager to examine any open Technology Change Requests (TCR) in a systematic manner. The meetings examine a TCRs impact on the project's risk, cost, schedule, and performance profile. The CCB ensures that all affected parties are cognizant of the changes and have a voice in the decision making process. The MSL Project Manager and MTP Program Manager chair the CCB and have authority to approve any changes which do not affect the projects Level 1 requirements. Advisor members of the CCB include the Technology Manager, the Element Managers, and the project Chief Engineer.

The MSL Technology Program maintains budget reserves to cover changes and other problems with the technology program. The lien list and the technology budget reserves are reviewed at the Change Control Board (CCB) meetings. The CCB balances the projects and the technology program's risk, cost, schedule, and performance. The CCB can decide to approve or disapprove a TCR. The CCB can also decide to keep the TCR open and review it again at the next CCB meeting. Projects are reluctant to spend reserves early in the fiscal year, but later in the year when potential problems are better understood, an existing lien may be approved.

In addition to reviewing changes, if a technology task is not performing due to cost, schedule, performance, or risk issues, the CCB can decide that the task's performance and priority do not warrant spending reserves. Another option is to cancel a technology task, adopt the current state-of-the-art performance, and fund a more promising effort on the technology lien list. The lien list and the different funding options provide a sense of urgency to the task managers to keep their tasks on track.

3.3.5 MTP Year-End Review (YER) - The MTP Program Manager organizes an October year-end review and assembles a peer review panel. The panel includes appropriate technical and programmatic personnel from JPL, NASA centers, NASA Headquarters, and universities. The MTP Program Manager or his designee chairs the peer review panel and provides a summary of review results and recommendations to the MSL Project Manager. 


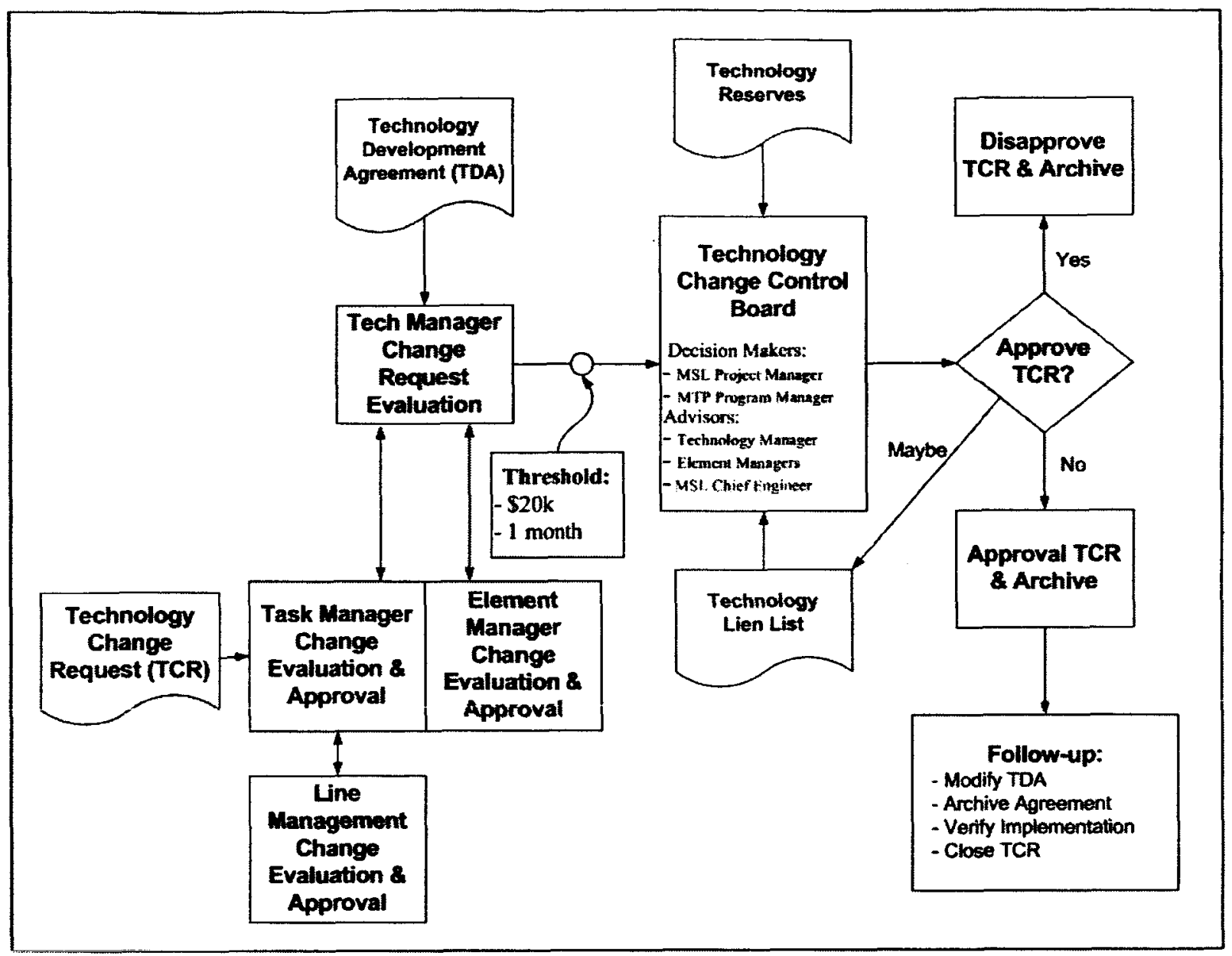

Figure 3-3 - The MSL Technology Change Control Flow

As mentioned in an earlier section, MTP used the 2002 year-end review to independently asses the MSL Technology Program and to serve as the final gate before the program transitioned into implementation.

3.3.6 Technology Readiness Certification Review Traditionally, a major issue with NASA technology programs is there in ability to successfully infuse technology into a flight project. To help solve this problem, the MSL technology program is a focused program and includes the MSL Project Team in the selection and implementation of the technologies. The initial infusion plans are also captured in the task's TDA.

In addition, to help accelerate and simplify the technology infusion process, MSL established a Technology Readiness Certification Review (TRCR) process. In this process, the Task Manager prepares a data package on their technology and presents the package to the project near the completion of the task. The package includes the criteria the task used to meet their TRL 6 requirements. For each technology, the TRCR package includes all the design, fabrication, assembly, and test information the project needs to implement the technology.

\section{TeChNOlogy Management ToOls}

The previous sections described the first year of the MSL technology program and examine the processes for defining, selecting, and managing the focused technology tasks. In addition to the processes defined above, the first year of the technology program also implemented a number of technology assessment tools. These tools are described in the following sections. A change in technology management occurred between the first and second year of the technology program and the tools were no longer implemented. The tools, however, were useful during the first year and warrant discussion.

\subsection{Aggregate Project Plan ("The Bubble Chart")}

The aggregate project plan (or as the Project Team called it, the Bubble Chart) maps the technical impact of each technology task against the risk of the task. The chart also includes the budget allocation and budget status of each task [5]. This chart allows managers to quickly assess the status of all technology tasks in MSL's Technology Program. 


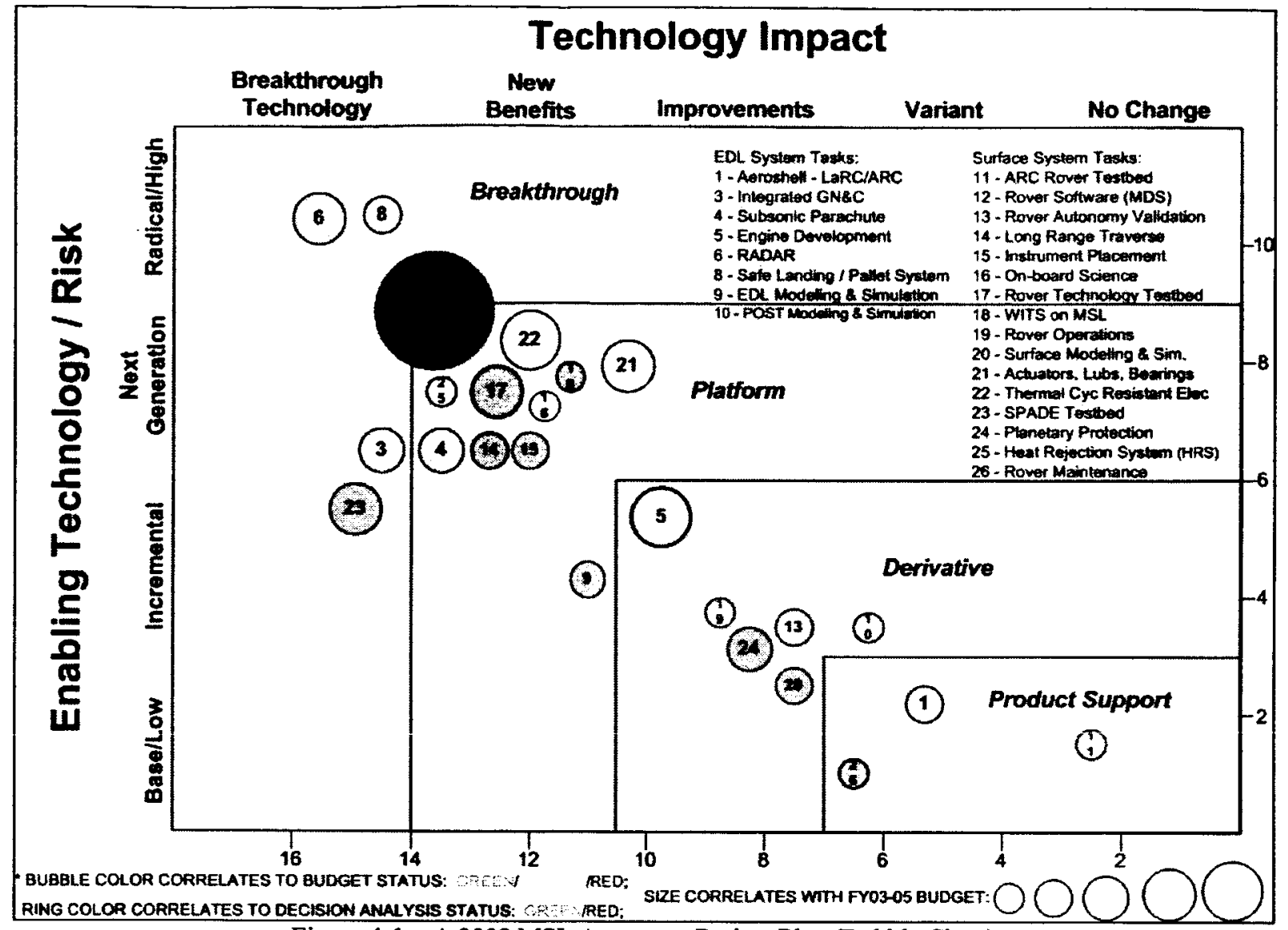

Figure 4-1 - A 2002 MSL Aggregate Project Plan (Bubble Chart)

A technology portfolio (represented by the aggregate project plan), like a financial portfolio, should be balanced. That is, the risk of developing a technology should be commensurate with the technology's predicted performance or impact. For example, a high-risk technology should have a high impact and a low impact technology better have a low development risk. In addition, if a technology has a high impact, but a low development risk, maybe it should be moved out of the technology program and into a flight project.

The aggregate project plan is updated every month with the latest status of the technology tasks. The bubble's $x / y$ location corresponds to the risk/impact of the technology; the size of the bubble corresponds to the task's budget, the color of the bubble corresponds to the task's financial status (red $=+/->10 \%$, yellow $=+/-5 \%$ to $10 \%$, green $=$ on budget); and the color of the bubble's ring corresponds to the status of the task's risk/decision analysis assessment. Figure 4-1 is an example of the MSL's technology aggregate project plan.

The process of creating the first MSL aggregate project plan (or the Bubble Chart), was a challenging process. Technologists don't like to compare their technologies headto-head to other technologies and want their technologies shown favorably in any summary chart. The $x / y$ location of each bubble was assign by its Task Manager and Element Manager, but some Task Managers disagree with their relationship to other tasks. The process is subjective and initiated some heated debates, but the end result is a useful management tool to access and correct the overall Technology Program.

For example, Figure 4-1 shows a high-impact, high-risk, task that was significantly over budget and had a budget status of red. This raised considerable management attention and both the Task Manager and Element Manager had to explain the issues and present a recovery plan. The recovery plan included reducing the future workforce, extending some delivery schedules, and using budget reserves to cover the overnuns. The project reviewed the plan, agreed the new schedule did not significantly impact the project, and approved the recovery plan. In addition, the one large task was broken into three smaller more manageable tasks.

The monthly aggregate project plan helped identify the problem, highlighted the impact and the risk of the task, and let management see the big picture before agreeing to a solution. If the task had a lower impact it may have been cancelled or not given more money. In addition, if the task had less risk (i.e. less challenging), but had similar problems, the Task Manager may have been replaced. 
The X-axis, or the Technology Impact, of the aggregate project plan is defined as following: 1) New Core Product Fills previously unknown, unmet needs. This is the first introduction of a revolutionary new technology. Stimulates new usage and system designs; 2) New Benefits - Fills known but as yet unmet needs. Delivers new concepts and benefits that fulfill needs that are otherwise unmet by any technology; 3) Improvement - Better meets needs or meets more needs. This impact is significant, discernible improvement or enhancement relative to existing technology; and 4) Variant - Meets same needs differently. This is a minor revision, adjustment, and alterations with relative parity to existing product performance, claims, \& features; and 5) No Change - This technology meets the same needs with no change. No perceived activity, but involving improved processes that result in cost reductions or meet project/program mandate with no change in quality.

The Y-axis, or the Risk, of the aggregate project plan is defined as following:: 1) Radical - First use of a Technology that is new to the industry; 2) Next Generation - Pushes existing Technologies into a completely different application; 3) Incremental - Extends existing Technologies beyond the normal application; and 4) Base - Exploits current standard Technology without extending the range of applications [5].

\subsection{Technology Funnel Chart}

The technology funnel chart maps the high-level project schedule against the schedule status of each the technology tasks (see Figure 4-2). It also captures the budget allocation and schedule status of each task. The funnel chart defines the separation between different technology development stages. The process provides a clear criteria for a technology to proceed to its next development step. It also provides a way to kill technology development efforts that is not performing technically, financially, or schedulewise. The funnel chart enables program and project managers to engage technologists at the right time to correct technical and programmatic issues before they impact the project. It also provides a continuous process to verify that the technology fits with the project and program strategy and system architecture. The overall shape of the funnel chart implies that some technology tasks are eliminated as the project refines its implementation concept and some technology tasks cannot meet their requirements [5].

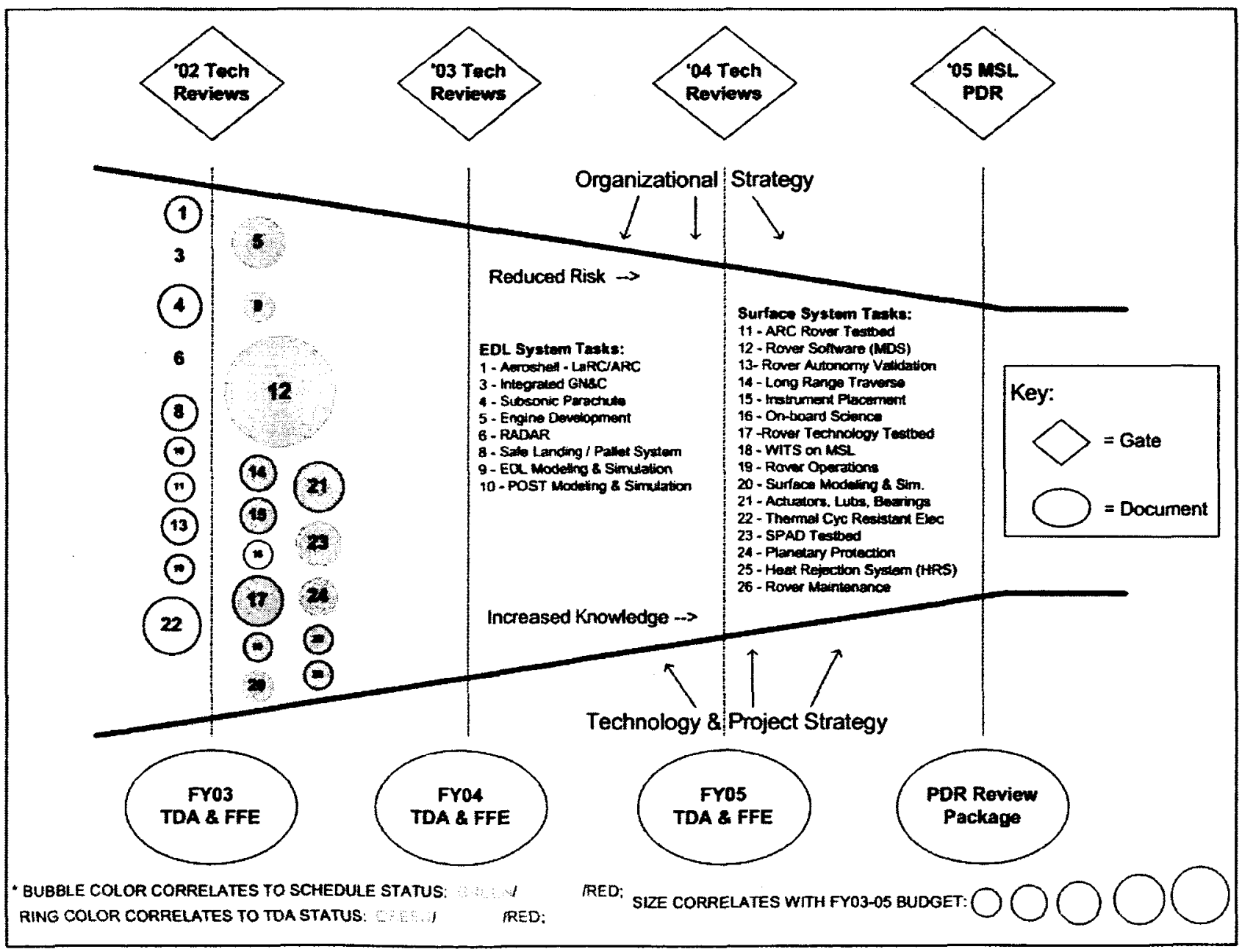

Figure 4-2 - A 2002 MSL Technology Funnel Chart 
The primary gates (represented by horizontal diamonds) on the funnel chart are the yearly non-advocate reviews and the quarterly program reviews. These gates give technologists the freedom they need between gates to develop their technologies. This phased gate model facilitates a common understanding of technology task progress. The gates allow the project to defined, track, and review tasks according to predetermined decision criteria. They also give a project visibility across tasks and elements with standard terminology and simplified reporting. The selection criteria for a task to pass from one phase of the funnel chart to the next include: are key project needs identified and met; will the technology be delivered within budget, schedule, and risk constraints; and does the technology fit the project architecture.

The ovals in the bottom of the funnel chart represent the documents the technology tasks develop to describe their tasks. These documents include the TDA and FFE, which reflect the detailed technical description of the task and the budget required to meet the technical requirements. The TDA and FFE's are periodically update to reflect the latest status of the task. Significant changes to the TDA and FFE require approval by the MSL technology CCB to properly assess the impact of any changes.

Figure 4-2 is an example of the MSL Project's technology funnel diagram. The funnel chart is updated every month with the latest status of the technology tasks. The bubble's $\mathrm{x}$-location corresponds to where the technology is on the TRL/progress scale; the size of the bubble corresponds to the task's budget, the color of the bubble corresponds to the task's schedule status; and the color of the bubble's ring corresponds to the technology's TDA status.

The funnel chart enables managers to quickly assess where technologies are in the development process and which technologies are behind schedule and require more management attention.

\subsection{Decision Analysis Tools}

The MSL Project uses a collection of Decision Analysis (DA) and Risk Assessment (RA) tools to manage the decisions and risks associated with MSL's technology development program. The decision tree captures and analyzes both the key decisions associated with each technology task and the key decisions that may impact other technologies and the project. A typical decision tree is in Figure 4-3 and the decision analysis associated with this figure was completed for the critical decisions associated with some of the technology development tasks.

Decision trees and the decision analysis process collects the critical decisions of the technology program; defines the performance, cost, and risk of each decision option; and establishes a date when the decision has to be made [7].
The decision analysis process ensures that the MSL Project makes intelligent and timely technology decisions.

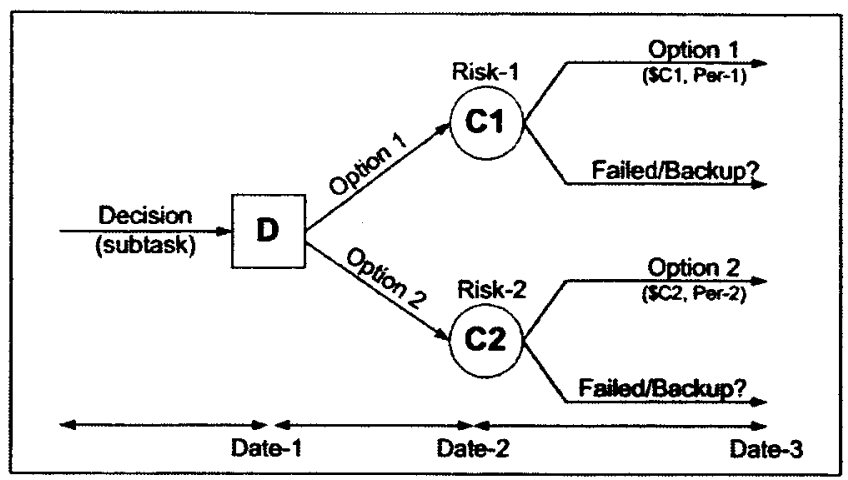

Figure 4-3 - The Decision Tree Analysis Tool

In addition to the decision analysis, the MSL technology tasks completed a risk assessment of the key technology deliverables. Figure 4-4 is an example of a deliverable's risk assessment. The performance, cost, risk, and date of each critical deliverable will be defined. If the risk of a technology's deliverable is high, a backup option may be defined. For these critical deliverables, the development schedules of the primary and backup options will be compared and if necessary, a parallel technology development effort may be initiated.

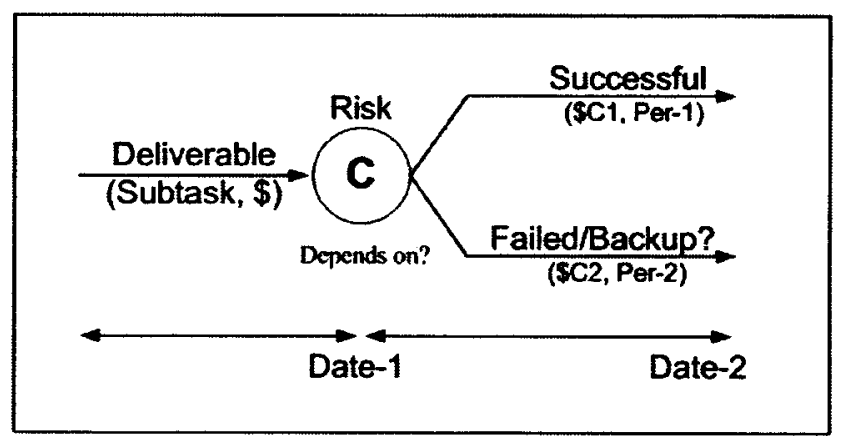

Figure 4-4 - The Deliverable Risk Analysis Tool

The task to modify the original Viking engines (used in the two 1976 Mars landings) is an example of using decision analysis. Early in the MSL Technology Program, the Project decided to modify the original Viking engine to increase performance rather than live with the current state of the art. The performance, risk, and cost of modifying the Viking engines, compared to the performance, risk, and cost of the current engines, warranted the new technology development effort. However, the project continued to monitor the deliverables of this task and if the task ever stumbled, the backup option and its impact on the mission concept would be considered. 


\section{CONCLUSION}

The MSL Technology Program is tightly coupled to the MSL mission and its milestones. The program develops critical deliverables that must be developed in time for infusion into the MSL mission. The plan is to reach TRL 6 for each technology by the mission's PDR. This program transcends the usual gulf between technology and projects by vertically integrating the technology work with preproject development in a project-like environment with critical dates for technology infusion. The program addresses developing key technology to enable MSL's revolutionary science mission.

\section{REFERENCES}

[1] Hayati, S., "The Mars Technology Program", Proceedings of the Joint Army-Navy-NASA-Air Force Propulsion Meeting, Orlando, Florida, Nov. 18-21, 2002.

[2] NASA Procedures and Guidelines, NPG 7120.5B, NASA Program and Project Management Processes and Requirements, November 21, 2002

[3] Cornford, S., Feather, M., Hicks, K., "DDP - A tool for life-cycle risk management", 2001 IEEE Aerospace Conference Proceedings

[4] Hayes, R.H., Wheelwright, S.C., and Clark, K.B. (1988). Dynamic Manufacturing. New York: The Free Press

[5] Henderson, R., (1999), Technology Management Lecture Notes, Mimeo, MIT 2002

[6] Franke, R.H. \& Kaul, J.D. "The Hawthorne experiments: First statistical interpretation." American Sociological Review, 1978, 43, 623-643.

[7] Apostolakis, G, (2000), Engineering Risk Benefit Analysis (ERBA) Lecture Notes, Mimeo, MIT 2002

\section{ACKNOWLEDGEMENTS}

Many people contributed to the successful MSL Technology Program and the authors would like to thank and acknowledge the following people: Michael Sander (MSL Project Manager); Matthew Golombek and Leslie Tamppari (Science); Charles Whetsel and Charles Weisbin (Mars Program); Curt Henry, Allen Chen, Richard Doyle, Stephen Dawson, Sam Thurman, and Rebecca Heninger (System Engineers); Brian Muirhead (Chief Engineer); Alice West (Resources); Margaret Easter (Procurement); Dave Woerner, Richard Volpe, Jeffery Umland, Jeffrey Simmons, and Sandy Krasner (Element Managers); Nancy Schweiner and Irene Lopez (Project Support); Susan Volk, Bruce MacNeal, and Carlos Balacuit (TDA Team); all the past and present MSL Task Managers (Lockwood, Wong, Carlson, Pollard, Rivellini, Balaram, Bualat, Lai, Peters, Huntsberger, W. S. Kim, Nesnas, Backes, Starbird, Jain, Johnson, Kolawa, Burke, Bolotin, Bearman, Kern, Birur, Baumgartner, Fesq, Page, Voorhees, Thoma, Laub, Mitcheltree, and Striepe); and Rebecca Henderson and Edward Crawley (MIT Sloan and Aero/Astro Professors).

\section{BIOGRAPHIES}

Mr. Robert Caffrey is the SEU Deputy Program Manager for Formulation at NASA GSFC. He has been at GSFC since September 1985 and spent the first eight years developing both flight hardware and software for the Shuttle Solar Backscatter Ultraviolet (SSBUV). Next, he moved to GSFC's Flight Data Systems Branch and developed the Essential Services Node

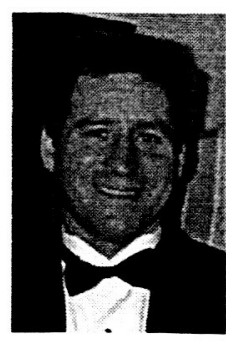
(ESN), a satellite data system 'on a chip'. He is a coinventor of the ESN and a patent holder for the ESN's ASIC. He then worked as the SeaWiFS Deputy Project Manager and then the SIMBIOS Deputy Project Manager. He was the Instrument Manager for VOLCAM, SEI, and ABI. He spent a year at JPL as MSL's Technology Manager. Mr. Caffrey earned two degrees from the University of Maryland - a co-op BS degree in Electrical Engineering (1985) and a BS degree in Computer Science (1990). Mr. Caffrey also earned a Master Degree at MIT's Sloan School of Management (2001) and was a MIT Research Affiliate until July 2002

Dr. Udomkesmalee received his $P h . D$. degree in Systems Science from University of California, San Diego and has over 20 years experience in line/project management, systems engineering, and control system design. As an employee of the Jet Propulsion Laboratory, Dr. Udomkesmalee has worked on a variety of

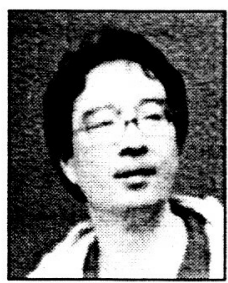
$R \& D$ and flight projects including AFAST, VIGILANTE, Galileo and Cassini. Currently, he manages the MSL Focused Technology program. Dr. Udomkesmalee has over 40 publications in the areas of spacecraft/missile guidance and control, GPS systems, celestial sensors, image processing and automatic target recognition.

Samad Hayati received his B.S. in Mechanical Engineering from Arya-Mehr University in Iran in 1971. He received M.S. and Ph.D. degrees in Controls from the University of California at Berkeley in 1972 and 1976, respectively. After holding the position of Assistant Professor of

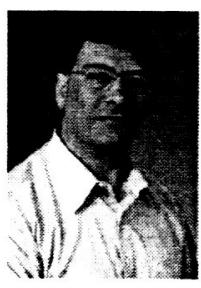
Mechanical Engineering at Arya-Mehr University, he joined NASA's Jet Propulsion Laboratory in 1979. He worked on the spacecraft Galileo from 1979 to 1983. He then joined the research group in robotics and pioneered a robot calibration technique that, in addition to industrial application, performed robot assisted brain surgery in 1986. In 1990 he led a team that developed the Rocky 7 rover and performed JPL's first remote field tests simulating Mars analog missions. Dr. Hayati has published over 50 articles and holds two US patents. In 1999 he was appointed Manager of NASA's Mars Technology Program, where he is responsible for the development of technologies to enable future NASA Mars missions. 